\title{
The drug-drug interaction between proton pump inhibitors and clopidogrel
}

\author{
Wei C. Lau MD, Paul A. Gurbel MD
}

$\infty$ See related research paper by Juurlink and colleagues, page 713

$\mathrm{T}$ he efficacy of clopidogrel in combination with acetylsalicylic acid (ASA) therapy has been clearly established in well-designed randomized controlled trials that have shown a reduction in recurrent coronary events following acute myocardial infarction, as compared with ASA monotherapy. ${ }^{1}$ However, the response to clopidogrel varies among patients, and clopidogrel resistance has been observed. Such variations in response have repeatedly been associated with adverse cardiovascular outcomes in patients undergoing percutaneous coronary intervention. ${ }^{2,3}$

The pharmacology of clopidogrel is key to understanding this phenomenon. Clopidogrel is a prodrug that must be converted to an active metabolite. The metabolite inhibits platelet aggregation (the rationale for clopidogrel's use in high-risk cardiovascular disease) by irreversibly binding to the platelet $\mathrm{P}_{2} \mathrm{Y}_{12}$ adenosine diphosphate receptor. In vivo, $85 \%$ of the clopidogrel dose is inactivated by plasma esterases. The remaining $15 \%$ is bioactivated in a 2-step pathway that depends on the cytochrome P450 isoenzyme system. The specific isoenzymes involved include cytochrome P450 1A2, 2B6, 2C9, 2C19 and 3A4. ${ }^{4}$ The cytochrome P450 2C19 and 3A4 isoenzymes play the major role. ${ }^{5}$

One mechanism for resistance to clopidogrel involves genetic polymorphisms that alter expression of cytochrome P450 isoenzymes that act on the drug. The result is insufficient enzymatic activity for optimal conversion of clopidogrel to its active metabolite. This mechanism is supported by recent data from the FAST-MI study (French Registry of Acute ST-Elevation and Non-ST-Elevation Myocardial Infarction study) and the TRITON-TIMI 38 study (Trial to Assess Improvement in Therapeutic Outcomes by Optimizing Platelet Inhibition with Prasugrel-Thrombolysis in Myocardial Infarction 38). Both of these studies demonstrated a greater than 3 -fold increase in the risk of adverse cardiovascular events among patients undergoing percutaneous coronary intervention who were homozygous or heterozygous for any of the CYP2C19 alleles known to result in a nonfunctional protein $(C Y P 2 C 19 * 2, * 3, * 4$ and $* 5)$, as compared with patients who had wild-type $C Y P 2 C 19 * 1$ allele. ${ }^{6,7}$

Another mechanism for clopidogrel resistance is competitive inhibition of cytochrome P450 isoenzymes needed for the metabolic activation of clopidogrel. Atorvastatin is a competitive inhibitor of the isoenzyme cytochrome P450 3A4. This interaction has been shown to have important effects on clopidogrel activity, as elucidated in small observational studies

\section{Key points}

- Clopidogrel requires bioactivation by cytochrome P450 isoenzymes in the liver in order to exert its inhibitory effect on platelet aggregation.

- Polymorphisms resulting in decreased enzymatic expression of cytochrome P450 2C19 and competitive inhibition of this isoenzyme by proton pump inhibitors impair activation of clopidogrel.

- Unlike other proton pump inhibitors, pantoprazole primarily inhibits cytochrome P450 2C9 and therefore causes less attenuation of the effect of clopidogrel.

- The concomitant use of proton pump inhibitors and clopidogrel should be limited to patients with sound clinical indications.

- Alternative treatment strategies with drugs not dependent on cytochrome P450 2C19 should be considered for multidrug therapy in patients at high risk for recurrent myocardial infarction.

involving patients undergoing percutaneous coronary intervention. ${ }^{8}$ Proton pump inhibitors are among the competitive inhibitors of cytochrome P450 2C19, the other major isoenzyme involved in the activation of clopidogrel. In a prospective, randomized, double-blind placebo-controlled study involving patients undergoing elective coronary artery stenting who received clopidogrel, co-administration of the proton pump inhibitor omeprazole was associated with decreased cytochrome P450 2C19-dependent inhibition of platelet aggregation (i.e., a decreased platelet inhibitory effect of clopidogrel). ${ }^{9}$

In this issue of $C M A J$, Juurlink and colleagues, using a population-based nested case-control study design, report on their investigation of the potential association of a cytochrome P450 2C19-dependent drug-drug interaction between clopidogrel and proton pump inhibitors and the risk of readmission to hospital because of myocardial infarction among patients 66 years or older who received clopidogrel therapy following hospital discharge after acute myocardial infarction. ${ }^{10}$ Patients who experienced reinfarction within 90 days after discharge were more likely than event-free patients

Wei Lau is Clinical Associate Professor, Director of Cardiovascular Thoracic Anesthesiology and Medical Director of the Cardiovascular Center Operating Rooms, University of Michigan Health System, Ann Arbor, USA. Paul Gurbel is Director of the Sinai Center for Thrombosis Research, Sinai Hospital of Baltimore, Baltimore, USA. 
in the control group to have received concomitant therapy with clopidogrel and proton pump inhibitors. The authors estimated that, compared with no treatment, cytochrome P450 2C19-inhibiting proton pump inhibitors were collectively associated with a $40 \%$ relative increase in the risk of recurrent myocardial infarction.

An exception was the proton pump inhibitor pantoprazole, which did not show the above associations. Juurlink and colleagues speculate as to why: unlike the other proton pump inhibitors (omeprazole, lansoprazole, rabeprazole and esomeprazole), pantoprazole exerts its highest inhibition potency toward cytochrome $\mathrm{P} 4502 \mathrm{C} 9$ rather than cytochrome $\mathrm{P} 4502 \mathrm{C} 19$, as has been demonstrated in vitro. ${ }^{11}$ As such, a drug-drug interaction leading to attenuation of the efficacy of clopidogrel is less likely with pantoprazole.

Juurlink and colleagues are to be commended for the comprehensiveness of their study. The study was robustly adjusted for the imbalances between the case and control groups in clinically important covariates, including cardiovascular comorbidities (including renal insufficiency, congestive heart failure and complications related to diabetes mellitus) and essential evidence-based cardiovascular protective medications (including angiotensin-converting-enzyme inhibitors, $\beta$ blockers and statins). However, limitations in addition to those discussed by the authors include lack of data on patient compliance with antiplatelet therapy. Also, although this study was not designed to examine the pharmacogenomics of proton pump inhibitors, it did not account for ethnicity, potentially relevant since genetic polymorphisms of cytochrome P450 2C19 leading to insufficient enzymatic activity are not equally distributed among individuals of white, Asian and African descent.

Induction of cytochrome P450 isoenzymes, leading to an enhanced platelet inhibitory effect of clopidogrel, has also been described, which suggests a means for overcoming clopidogrel resistance. Hyperforin, a constituent of St. John's wort, induces metabolic activity of cytochrome P450 2C19 and cytochrome P450 3A4 in the liver. As such, it increases the effect of drugs that depend on these 2 isoenzymes for their metabolic activation. ${ }^{12}$ In a small prospective study, administration of St. John's wort enhanced the platelet inhibitory effect of clopidogrel in volunteers known to be unresponsive to the drug and in patients with stable coronary artery disease. ${ }^{13}$

Understanding drug interactions that impair therapeutic efficacy is important, especially with multidrug treatment, as exemplified by the patient cohort with high-risk cardiovascular disease in the study by Juurlink and colleagues. Although their findings are provocative, definitive evidence of the effect of proton pump inhibitors on the antiplatelet properties of clopidogrel would require large prospective studies. Future studies designed to investigate the relation of proton pump inhibitors to adverse cardiovascular outcomes should strive to adjust for imbalances in cardiovascular-related comorbidities, patient ethnicity and concomitant drug use between patient groups, and carefully measure patient compliance with therapy.
However, given the observational data presented thus far, a future randomized clinical trial to determine increased cardiovascular risk associated with proton pump inhibitors is unlikely. At this point, concomitant therapy with a cytochrome P450 2C19-inhibiting proton pump inhibitor and clopidogrel should be administered when there is a sound clinical indication. For example, patients taking clopidogrel and warfarin therapy who require a proton pump inhibitor may need to avoid pantoprazole, since warfarin is metabolized primarily by cytochrome P450 2C9. Alternatively, treatment strategies may be considered that use drugs not dependent on the cytochrome $\mathrm{P} 4502 \mathrm{C} 19$ isoenzyme, such as pantoprazole and $\mathrm{H}_{2}-$ receptor antagonists.

Competing interests: Wei Lau has received a research grant from Eli Lilly for study of the drug-drug interaction of clopidogrel and simvastatin. Paul Gurbel has received honoraria, research grants and consultant fees from AstraZeneca, Schering-Plough, Portola Pharmaceuticals, Bayer, Daiichi Sankyo and Eli Lilly.

Contributors: Both of the authors were involved in the preparation of the manuscript and approved the final version submitted for publication.

\section{REFERENCES}

1. Yusuf S, Zhao F, Mehta SR, et al.; Clopidogrel in Unstable Angina to Prevent Recurrent Events Trial Investigators. Effects of clopidogrel in addition to aspirin in patients with acute coronary syndromes without ST-segment elevation. $N$ Engl J Med 2001;345:494-502.

2. Gurbel PA, Bliden KP, Samara W, et al. Clopidogrel effect on platelet reactivity in patients with stent thrombosis: results of the CREST Study. J Am Coll Cardiol 2005;46:1827-32.

3. Bliden KP, DiChiara J, Tantry US, et al. Increased risk in patients with high platelet aggregation receiving chronic clopidogrel therapy undergoing percutaneous coronary intervention: Is the current antiplatelet therapy adequate? $\mathrm{J} \mathrm{Am}$ Coll Cardiol 2007;49:657-66.

4. Farid NA, Payne CD, Small DS, et al. Cytochrome P450 3A inhibition by ketoconazole affects prasugrel and clopidogrel pharmacokinetics and pharmacodynamics differently. Clin Pharmacol Ther 2007;81:735-41.

5. Kim KA, Park PW, Hong SJ, et al. The effect of $C Y P 2 C 19$ polymorphism on the pharmacokinetics and pharmacodynamics of clopidogrel: a possible mechanism for clopidogrel resistance. Clin Pharmacol Ther 2008;84:236-42.

6. Simon T, Verstuyft C, Mary-Krause M, et al.; French Registry of Acute ST-Elevation and Non-ST-Elevation Myocardial Infarction (FAST-MI) Investigators. Genetic determinants of response to clopidogrel and cardiovascular events. $N$ Engl J Med 2009;360:363-75.

7. Mega JL, Close SL, Wiviott SD, et al. Cytochrome p-450 polymorphisms and response to clopidogrel. N Engl J Med 2009;360:354-62.

8. Lau WC, Waskell LA, Watkins PB, et al. Atorvastatin reduces the ability of clopidogrel to inhibit platelet aggregation: a new drug-drug interaction. Circulation 2003; 107:32-7.

9. Gilard M, Arnaud B, Cornily JC, et al. Influence of omeprazole on the antiplatelet action of clopidogrel associated with aspirin: the randomized, double-blind OCLA (Omeprazole CLopidogrel Aspirin) study. J Am Coll Cardiol 2008;51:256-60.

10. Juurlink DN, Gomes T, Ko DT, et al. A population-based study of the drug interaction between proton pump inhibitors and clopidogrel. CMAJ 2009;180:713-8.

11. Li XQ, Andersson TB, Ahlström M, et al. Comparison of inhibitory effects of the proton pump-inhibiting drugs omeprazole, esomeprazole, lansoprazole, pantoprazole, and rabeprazole on human cytochrome P450 activities. Drug Metab Dispos 2004;32:821-7.

12. Wang LS, Zhu B, Abd El-Aty AM, et al. The influence of St John's Wort on CYP2C19 activity with respect to genotype. J Clin Pharmacol 2004;44:577-81.

13. Lau WC, Gurbel P, Bates ER. St. John's Wort enhances the platelet inhibitory effect of clopidogrel in clopidogrel "resistant" healthy volunteers [abstract]. J Am Coll Cardiol 2005;45(Suppl A):382A.

Correspondence to: Dr. Wei C. Lau, 4172 Cardiovascular Center, 1500 E Medical Center Dr., SPC 5861, Ann Arbor MI 48109-5861, USA; fax 734 936-9091; weiclau@med.umich.edu 